\title{
Oligochaeta (Annelida: Clitellata) associated to aquatic macrophytes in Brazil
}

\author{
Nathalie Aparecida de Oliveira Sanches ${ }^{1 *}$ \\ Marina Gulo Alcorinte ${ }^{2}$ \\ Lucas Henrique Sahm ${ }^{1}$ \\ Guilherme Rossi Gorni ${ }^{1}$ \\ Maria Lúcia Ribeiro ${ }^{1}$ \\ Centro Universitário de Araraquara \\ ${ }^{1}$ Programa de Pós-Graduação em Desenvolvimento Territorial e Meio Ambiente \\ ${ }^{2}$ Curso de Graduação em Ciências Biológicas, Departamento de Ciências Biológicas e da Saúde \\ Rua Carlos Gomes, 1338, Centro, CEP 14801-340, Araraquara - SP, Brasil \\ * Autor para correspondência \\ nathalie_sanches@hotmail.com
}

Submetido em 16/10/2015

Aceito para publicação em 02/05/2016

\section{Resumo}

Oligochaeta (Annelida: Clitellata) associados a macrófitas aquáticas no Brasil. Oligoquetos ainda são caracterizados como um grupo pouco estudado dentre os macroinvertebrados aquáticos e poucos estudos sobre sua ecologia foram realizados no Brasil. Assim, nosso estudo objetivou fornecer um panorama da associação entre Oligochaeta e macrófitas, em ambientes aquáticos continentais brasileiros, por meio de uma revisão da literatura junto a um inventário de espécies associadas a macrófitas aquáticas em lagoas marginais da represa Ribeirão das Anhumas (Américo Brasiliense-SP). Na revisão, analisamos 10 artigos, nos quais obtivemos dados sobre 41 espécies. Amostramos, ainda, cinco gêneros de macrófitas, Egeria, Salvinia, Utricularia, Eleocharis e Ceratophyllum, em agosto e dezembro de 2012 e em março e abril de 2013, na represa Ribeirão das Anhumas. Registramos 21 espécies de Oligochaeta associadas a essas macrófitas. Com os dados obtidos na revisão junto ao inventário da represa Ribeirão das Anhumas, evidenciamos o total de 41 espécies associadas a macrófitas aquáticas, com maior riqueza da família Naididae (93,33\%), seguida por Opistocystidae (4,44\%) e Alluroididae $(2,22 \%)$. Nosso estudo inventariou cerca de $48 \%$ da diversidade de Oligochaeta registrados em ecossistemas continentais no Brasil, destacando, assim, a importância das macrófitas como recurso para esses invertebrados, principalmente para a família Naididae.

Palavras-chave: Ecossistemas de água doce; Fauna associada; Lista de espécies; Microdrili

\section{Abstract}

Oligochaeta are still characterized as a poorly studied group among the aquatic macroinvertebrates and few studies about their ecology were conducted in Brazil. Thus, our study aimed to provide an overview of the association between Oligochaeta and macrophytes, in Brazilian continental aquatic environments, by means of a literature review along with an inventory of species associated to aquatic macrophytes on marginal lagoons 
in the reservoir Ribeirão das Anhumas (Américo Brasiliense, São Paulo, Brazil). In the review, we analyzed 10 articles, where we obtained data on 41 species. We also sampled 5 macrophyte genera, Egeria, Salvinia, Utricularia, Eleocharis, and Ceratophyllum, in August and December 2012 and in March and April 2013, in the reservoir Ribeirão das Anhumas. We registered 21 Oligochaeta species associated to these macrophytes. With the data obtained in the review along with the inventory of the reservoir Ribeirão das Anhumas, we found a total of 41 species associated to aquatic macrophytes, with a higher richness of the Naididae family $(93.33 \%)$, followed by Opistocystidae (4.44\%), and Alluroididae (2.22\%). Our study inventoried about $48 \%$ of the Oligochaeta diversity registered in continental ecosystems in Brazil, thus highlighting the significance of macrophytes as a resource for these invertebrates, mainly for the Naididae family.

Key words: Associated fauna; Freshwater ecosystems; Microdrili; Species list

\section{Introduction}

Research relying on information obtained from inventories of species provides a broad knowledge about distribution, richness, and other ecological aspects, establishing relevant tools for biodiversity conservation programs and decision-making with regard to management of natural areas (SILVEIRA et al., 2010). However, in general, the invertebrate fauna is still poorly documented when compared to other animal groups, such as mammals and birds (CHRISTOFFERSEN, 2010). The difficulties of taxonomic identification and the low popularity of these taxa among people make information about them fragmented and scarce (MAGURRAN, 2011).

Thus, one of the most abundant classes among aquatic macroinvertebrates, the Oligochaeta, is a relevant representative of the aquatic fauna, participating in the decomposition of organic matter and the cycling process of freshwater ecosystems (ESTEVES et al., 2011). In continental aquatic environments, Christoffersen (2007) catalogued 171 Oligochaeta species in South America, out of which 86 occur in Brazil. Moreover, Oligochaeta can be found in many kinds of substrates, such as sandy, clayey (DORNFELD et al., 2006; ALVES et al., 2008; PETSCH et al., 2013; 2015), or enriched organic matter (BEHREND et al., 2012). Oligochaeta are also associated to other organisms, such as bryophytes (GORNI; ALVES, 2007), sponges (GORNI; ALVES, 2008), molluscs (GORNI; ALVES, 2006), amphibians (ODA et al., 2015) and aquatic macrophytes (CORREIA; TRIVINHO-STRIXINO, 1998; TRIVINHO-STRIXINO et al., 2000; ALVES; GORNI, 2007).
Aquatic macrophytes are important elements to maintain biodiversity in tropical lakes, increasing habitat complexity and heterogeneity (ROSINE, 1955). Aquatic macrophytes are key in the trophic chain, where many animal groups obtain their food from biomass, organic residue, or periphyton growing on the surface of macrophytes (WELCH, 1952; ROSINE, 1955; POTT; POTT, 2000). Moreover, macrophytes serve as cover for excessive luminosity (WELCH, 1952), shelter against predators, and they can be used as nest for egg deposition (GLOWACKA et al., 1976). Invertebrate density and biomass may change according to the habitat complexity degree, which is optimized by macrophytes (MORMUL et al., 2010; KRAWCZYK et al., 2013).

Thus, Oligochaeta are often observed in areas with aquatic vegetation, mainly on decomposing leaves, where they feed on organic matter, as well as on periphyton adhered to the surface (GALIZZI; MARCHESE, 2009; MARTINS et al., 2011). Periphyton is a major source of food for Oligochaeta, especially the Naididae family (LEARNER et al., 1978). Thus, morphological plant structures serve as filters to catch particles and microorganisms, providing more favorable conditions so that Oligochaeta populations can establish and grow with macrophytes (CORREIA; TRIVINHO-STRIXINO, 1998). Moreover, plant structures are important to provide these organisms with shelter (CHAUVET, 1997), mainly submerged roots (ARMENDÁRIZ, 2008).

Taxonomic keys focused on Neotropical Oligochaeta (RIGHI, 1984; BRINKHURST; MARCHESE, 1989) have boosted development of many studies (MONTANHOLI-MARTINS; TAKEDA, 2001; PAMPLIN et al., 2005; ALVES et al., 2006; 2008; GORNI; ALVES, 2012; RODRIGUES et 
al., 2013; GORNI et al., 2015), which has been expanding knowledge about taxonomic and ecological Brazilian worms aspects. Moreover, in recent years the Oligochaeta class has been gaining attention, especially in biomonitoring and environmental ecotoxicology researches, on which it is use as pollution bioindicator and test organism (CHAPMAN, 2001; CORBI et al., 2015), due to its cosmopolitan distribution; numerical abundance; limited mobility and suitability for use in laboratory studies (HELLAWELL, 1986).

Despite the growing interest in studying and expand the ecological and biological knowledge of Oligocaheta, this research area still fragmented and incomplete in Brazilian freshwaters (ALVES et al., 2006). Based on the above, we aimed to inventory the Oligochaeta species associated to macrophytes in Brazilian continental aquatic environments.

\section{Material and Methods}

\section{Data from articles}

We searched Brazilian articles about the association between Oligochaeta and macrophytes in March and April of 2014 on the databases Scopus, Google Scholar, and Web of Science, by using the keywords "aquatic macrophytes" and "Oligochaeta" with no determined period. A total of 10 articles were selected according to the criterion of having Oligochaeta species or genus associated to macrophyte species or genus and we filtered only articles from Brazil. The articles found provide us with an overview from 1997 to 2014. To organize the results, a table was prepared according to the taxonomic level adopted (species or genus).

\section{Sampling of Oligochaeta}

We obtained information to inventory Oligochaeta on marginal lagoons in the reservoir Ribeirão das Anhumas (Américo Brasiliense, São Paulo, Brazil), central region of the state of São Paulo, Brazil (21\%42'23"S and 48ㅇ'33'W; $544 \mathrm{~m}$ high). Macrophytes were sampled in August and December 2012 and in March and April 2013 , on the coastal region of lagoons. According to
Pott and Pott (2000), we selected and identified five macrophyte genera: Egeria sp. (Hydrocharitaceae), Salvinia sp. (Salvinaceae), Utricularia sp. (Lentibulariaceae), Eleocharis sp. (Cyperaceae), and Ceratophyllum sp. (Ceratophyllaceae).

We collected $100 \mathrm{~g}$ (wet weight) for each macrophyte genus by using a $0.21 \mathrm{~mm}$ mesh hand net. Small amounts of each macrophyte were analyzed in trays with water and each Oligochaeta specimen was fixed in $10 \%$ formalin and preserved in $70 \%$ alcohol (ALVES; GORNI, 2007; PEIRÓ; GORNI, 2010). For identifying Oligochaeta, lamina were prepared with lactophenol and observed criteria adopted by Brinkhurst and Jamieson (1971), Righi (1984), Brinkhurst and Marchese (1989).

\section{Results}

We inventoried 21 Oligochaeta species on the lagoons in the reservoir Ribeirão das Anhumas, belonging to two families: Naididae and Opistocystidae. Sampling results were entered and 45 taxa were registered (41 species and 4 genera), associated to 20 aquatic macrophyte taxa (Table 1). The articles found included few Brazilian states: Mato Grosso do Sul, São Paulo, Paraná, and Minas Gerais, showing a concentration of studies in the southeastern, southern, and central-western regions in Brazil. Additionally, only one article discussed the occurrence of Oligochaeta on decomposing leaves (MARTINS et al., 2011), showing that most studies still focus on the sampling of living macrophytes.

Therefore, we noticed a predominance of the Naididae family among the species inventoried (literature review and inventory of species), with $93.33 \%$ associated to macrophytes and other two families registered: Opistocystidae (4.44\%) and Alluroididae $(2.22 \%)$. Moreover, the species with the highest prevalence in the macrophytes was Dero nivea, which was associated to 15 macrophyte taxa, followed by Dero digitata and Dero raviensis, associated to 11 taxa, and Dero sawayai, associated to 10 taxa. In turn, species such as Brinkhurstia americana and Limnodrilus hoffmesteiri were registered only in 1 macrophyte taxon. 
The most frequently registered macrophytes in literature review and inventory of species live a submerged life. However, the macrophyte showing the highest richness of Oligochaeta associated was
Eichhornia azurea, which lives a floating life. Thus, $E$. azurea had $51.1 \%$ of the richness, while the others were: Egeria sp. (40.0\%), Najas sp. (35.6\%), and Mayaca fluviatilis (31.1\%) (Table 2).

TABLE 1: Occurrence of Oligochaeta with aquatic macrophytes in Brazil.

\begin{tabular}{|c|c|c|}
\hline Oligochaeta & Aquatic macrophytes & References \\
\hline \multicolumn{3}{|l|}{ Alluroididae } \\
\hline $\begin{array}{l}\text { Brinkhurstia americana } \\
\text { (Brinkhurst, 1964) }\end{array}$ & Eichhornia azurea & $\begin{array}{l}\text { Montanholi-Martins and } \\
\text { Takeda (2001) }\end{array}$ \\
\hline \multicolumn{3}{|l|}{ Naididae } \\
\hline \multirow{2}{*}{ Allonais chelata (Marcus, 1944) } & Mayaca sp.; Najas sp.; Utricularia sp. & Alves and Gorni (2007) \\
\hline & Ceratophyllum sp. & This study \\
\hline \multirow{2}{*}{$\begin{array}{l}\text { Allonais inaequalis (Stephenson, } \\
\text { 1911) }\end{array}$} & Potamogeton sp.; Ipomoea sp.; Eleocharis sp.; Najas sp. & Alves and Gorni (2007) \\
\hline & Mayaca fluviatilis; Najas sp. & Gorni and Alves (2008) \\
\hline \multirow{4}{*}{$\begin{array}{l}\text { Allonais paraguayensis } \\
\text { (Michaelsen, 1905) }\end{array}$} & Scirpus cubensis & $\begin{array}{l}\text { Correia and Trivinho- } \\
\text { Strixino (1998) }\end{array}$ \\
\hline & $\begin{array}{c}\text { Hydrilla verticillata } \\
\end{array}$ & Behrend et al. (2013) \\
\hline & $\begin{array}{c}\text { Scirpus cubensis; Cabomba piauhyensis; Utricularia sp.; } \\
\text { Salvinia auriculata }\end{array}$ & $\begin{array}{l}\text { Trivinho-Strixino et al. } \\
\qquad(2000)\end{array}$ \\
\hline & $\begin{array}{l}\text { Salvinia } \text { sp. } \\
\end{array}$ & This study \\
\hline \multirow{2}{*}{$\begin{array}{l}\text { Aulodrillus pigueti Kowalewski, } \\
1914\end{array}$} & Polygonum sp.; Salvinia sp.; Eichhornia azurea & $\begin{array}{l}\text { Montanholi-Martins and } \\
\text { Takeda (2001) }\end{array}$ \\
\hline & Ceratophyllum sp.; Egeria sp.; Eleocharis sp. & This study \\
\hline $\begin{array}{l}\text { Aulophorus bimagnasetus Harman, } \\
1974\end{array}$ & Eleocharis sp.; Nymphaea sp.; Utricularia sp. & $\begin{array}{l}\text { Campitelli-Ramos et al. } \\
\text { (2014) }\end{array}$ \\
\hline \multirow{3}{*}{$\begin{array}{l}\text { Aulophorus borelii (Michaelsen, } \\
\text { 1900) }\end{array}$} & Scirpus cubensis & $\begin{array}{l}\text { Correia and Trivinho- } \\
\text { Strixino (1998) }\end{array}$ \\
\hline & Scirpus cubensis & $\begin{array}{l}\text { Trivinho-Strixino et al. } \\
\qquad(2000)\end{array}$ \\
\hline & Egeria sp.; Salvinia sp. & This study \\
\hline \multirow{2}{*}{$\begin{array}{l}\text { Aulophorus carteri Stephenson, } \\
1931\end{array}$} & Scirpus cubensis & $\begin{array}{l}\text { Correia and Trivinho- } \\
\text { Strixino (1998) }\end{array}$ \\
\hline & Scirpus cubensis; Utricularia sp. & $\begin{array}{c}\text { Trivinho-Strixino et al. } \\
(2000)\end{array}$ \\
\hline \multirow{3}{*}{$\begin{array}{l}\text { Aulophorus costatus Du Bois- } \\
\text { Reymond Marcus, } 1944\end{array}$} & Najas sp. & Alves and Gorni (2007) \\
\hline & Mayaca fluviatilis; Najas sp. & Gorni and Alves (2008) \\
\hline & Salvinia sp.; Ceratophyllum sp.; Egeria sp. & This study \\
\hline \multirow{2}{*}{$\begin{array}{l}\text { Aulophorus furcatus (O. F. Müller, } \\
\text { 1774) }\end{array}$} & Eichhornia azurea; Salvinia sp. & $\begin{array}{l}\text { Montanholi-Martins and } \\
\text { Takeda (2001) }\end{array}$ \\
\hline & Eichhornia azurea & Martins et al. (2011) \\
\hline \multirow{2}{*}{$\begin{array}{l}\text { Aulophorus lodeni (Brinkhurst, } \\
\text { 1986) }\end{array}$} & Scirpus cubensis & $\begin{array}{l}\text { Correia and Trivinho- } \\
\text { Strixino (1998) }\end{array}$ \\
\hline & $\begin{array}{c}\text { Eichhornia azurea; Salvinia auriculata; Scirpus } \\
\text { cubensis }\end{array}$ & $\begin{array}{l}\text { Trivinho-Strixino et al. } \\
(2000)\end{array}$ \\
\hline Aulophorus sp. Schmarda, 1861 & $\begin{array}{c}\text { Cabomba sp.; Cerathophylum sp.; Eichhornia azurea; } \\
\text { Scirpus sp. }\end{array}$ & $\begin{array}{c}\text { Trivinho-Strixino et al. } \\
(1997)\end{array}$ \\
\hline $\begin{array}{l}\text { Bratislavia dadayi (Michaelsen, } \\
\text { 1905) }\end{array}$ & Eichhornia azurea; Polygonum sp. & $\begin{array}{l}\text { Montanholi-Martins and } \\
\text { Takeda (2001) }\end{array}$ \\
\hline
\end{tabular}




\begin{tabular}{|c|c|c|}
\hline \multirow{3}{*}{$\begin{array}{l}\text { Chaetogaster diaphanus } \\
\text { (Gruithuisen, 1828) }\end{array}$} & Mayaca fluviatilis; Najas sp. & Gorni and Alves (2008) \\
\hline & Eichhornia azurea & Martins et al. (2011) \\
\hline & Egeria sp.; Salvinia sp.; Utricularia sp. & This study \\
\hline \multirow{3}{*}{$\begin{array}{l}\text { Chaetogaster diastrophus } \\
\text { (Gruithuisen, 1828) }\end{array}$} & Mayaca fluviatilis; Najas sp. & Gorni and Alves (2008) \\
\hline & Eichhornia azurea & Martins et al. (2011) \\
\hline & Hydrilla verticillata; Egeria najas & Behrend et al. (2013) \\
\hline \multirow{3}{*}{ Dero digitata (O. F. Müller,1773) } & $\begin{array}{l}\text { Egeria sp.; Cabomba sp.; Potamogeton sp.; Ipomoea sp.; } \\
\text { Eleocharis sp.; Mayaca sp; Utricularia sp.; Najas sp. }\end{array}$ & Alves and Gorni (2007) \\
\hline & Hydrilla verticillata; Egeria najas & Behrend et al. (2013) \\
\hline & Egeria sp.; Salvinia sp. & This study \\
\hline \multirow{3}{*}{ Dero evelinae Marcus, 1943} & Scirpus cubensis & $\begin{array}{l}\text { Correia and Trivinho- } \\
\text { Strixino (1998) }\end{array}$ \\
\hline & $\begin{array}{c}\text { Scirpus cubensis; Cabomba piauhyensis; Eichhornia } \\
\text { azurea; Salvinia auriculata }\end{array}$ & $\begin{array}{c}\text { Trivinho-Strixino et al. } \\
(2000)\end{array}$ \\
\hline & Potamogeton $\mathrm{sp}$. & Alves and Gorni (2007) \\
\hline \multirow{5}{*}{ Dero nivea Aiyer, 1930} & Scirpus cubensis & $\begin{array}{l}\text { Correia and Trivinho- } \\
\text { Strixino (1998) }\end{array}$ \\
\hline & $\begin{array}{c}\text { Scirpus cubensis; Eichhornia azurea; Cabomba } \\
\text { piauhyensis; Utricularia sp.; Salvinia auriculata }\end{array}$ & $\begin{array}{l}\text { Trivinho-Strixino et al. } \\
\text { (2000) }\end{array}$ \\
\hline & $\begin{array}{l}\text { Egeria sp.; Cabomba sp.; Potamogeton sp.; Ipomoea sp.; } \\
\text { Eleocharis sp.; Mayaca sp.; Utricularia sp.; Najas sp. }\end{array}$ & Alves and Gorni (2007) \\
\hline & Mayaca fluviatilis; Najas sp. & Gorni and Alves (2008) \\
\hline & Egeria sp.; Salvinia sp.; Ceratophyllum sp. & This study \\
\hline \multirow{2}{*}{ Dero obtusa D’Udekem, 1855} & Cabomba sp.; Eleocharis sp.; Ipomoea sp.; Najas sp. & Alves and Gorni (2007) \\
\hline & Mayaca fluviatilis; Najas $\mathrm{sp.}$ & Gorni and Alves (2008) \\
\hline Dero pectinata Aiyer, 1930 & Eichhornia azurea & $\begin{array}{l}\text { Montanholi-Martins and } \\
\text { Takeda (2001) }\end{array}$ \\
\hline \multirow{3}{*}{ Dero raviensis (Stephenson, 1914) } & $\begin{array}{l}\text { Egeria sp.; Cabomba sp.; Potamogeton sp.; Ipomoea sp.; } \\
\text { Eleocharis sp.; Mayaca sp.; Utricularia sp.; Najas sp. }\end{array}$ & Alves and Gorni (2007) \\
\hline & Mayaca fluviatilis; Najas sp. & Gorni and Alves (2008) \\
\hline & Ceratophyllum sp. & This study \\
\hline Dero righii Varela, 1990 & Eichhornia azurea & $\begin{array}{l}\text { Montanholi-Martins and } \\
\text { Takeda (2001) } \\
\end{array}$ \\
\hline \multirow{5}{*}{ Dero sawayai Marcus, 1943} & $\begin{array}{c}\text { Egeria sp.; Potamogeton sp.; Ipomoea sp.; Eleocharis sp.; } \\
\text { Najas sp. }\end{array}$ & Alves and Gorni (2007) \\
\hline & $\begin{array}{c}\text { Mayaca fluviatilis; Najas sp. } \\
\end{array}$ & Gorni and Alves (2008) \\
\hline & Eichhornia azurea & Martins et al. (2011) \\
\hline & Hydrilla verticillata; Egeria najas & Behrend et al. (2013) \\
\hline & Egeria sp.; Utricularia sp. & This study \\
\hline \multirow{3}{*}{ Dero sp. Oken, 1815} & $\begin{array}{c}\text { Cabomba sp.; Cerathophylum sp.; Scirpus sp.; } \\
\text { Eichhornia azurea }\end{array}$ & $\begin{array}{l}\text { Trivinho-Strixino et al. } \\
\text { (1997) }\end{array}$ \\
\hline & Hydrilla verticillata; Egeria najas & Behrend et al. (2013) \\
\hline & Eichhornia azurea & $\begin{array}{l}\text { Saulino and Trivinho- } \\
\text { Strixino (2014) }\end{array}$ \\
\hline $\begin{array}{l}\text { Limnodrilus hoffmeisteri } \\
\text { (Claparède, 1862) }\end{array}$ & Ceratophyllum sp. & This study \\
\hline \multirow{4}{*}{ Nais communis Piguet, 1906} & Cabomba piauhyensis & Trivinho-Strixino et al. (2000) \\
\hline & Ipomoea sp.; Najas sp. & Alves and Gorni (2007) \\
\hline & Mayaca fluviatilis; Najas sp. & Gorni and Alves (2008) \\
\hline & Hydrilla verticillata; Egeria najas & Behrend et al. (2013) \\
\hline Nais variabilis Piguet, 1906 & Cabomba sp. & Alves and Gorni (2007) \\
\hline
\end{tabular}




\begin{tabular}{|c|c|c|}
\hline $\begin{array}{l}\text { Paranadrilus descolei Gavrilov, } \\
1955\end{array}$ & Eichhornia azurea; Polygonum sp. & $\begin{array}{c}\text { Montanholi-Martins and } \\
\text { Takeda (2001) } \\
\end{array}$ \\
\hline \multirow{2}{*}{ Pristina aequiseta Bourne, 1891} & Mayaca fluviatilis; Najas sp. & Gorni and Alves (2008) \\
\hline & Eichhornia azurea & Martins et al. (2011) \\
\hline \multirow{3}{*}{$\begin{array}{l}\text { Pristina americana Cernosvitov, } \\
1937\end{array}$} & Eichhornia azurea; Polygonum sp. & $\begin{array}{c}\text { Montanholi-Martins and } \\
\text { Takeda (2001) }\end{array}$ \\
\hline & Eichhornia azurea & Martins et al. (2011) \\
\hline & Egeria najas & Behrend et al. (2013) \\
\hline \multirow{4}{*}{ Pristina biserrata Chen, 1940} & Eleocharis sp.; Potamogeton sp. & Alves and Gorni (2007) \\
\hline & Mayaca fluviatilis; Najas sp. & Gorni and Alves (2008) \\
\hline & Eichhornia azurea & Martins et al. (2011) \\
\hline & Ceratophyllum sp.; Egeria sp.; Salvinia sp. & This study \\
\hline Pristina breviseta Bourne, 1891 & $\begin{array}{l}\text { Egeria } \mathrm{sp} . \\
\end{array}$ & This study \\
\hline \multirow{4}{*}{ Pristina longiseta Ehrenberg, 1828} & Egeria sp.; Ipomoea sp.; Najas sp. & Alves and Gorni (2007) \\
\hline & Mayaca fluviatilis; Najas sp. & Gorni and Alves (2008) \\
\hline & Eichhornia azurea & Martins et al. (2011) \\
\hline & Ceratophyllum sp.; Salvinia $\mathrm{sp} . ;$ Egeria $\mathrm{sp}$. & This study \\
\hline \multirow{3}{*}{$\begin{array}{l}\text { Pristina macrochaeta Stephenson, } \\
1931\end{array}$} & Eleocharis sp.; Ipomoea sp.; Potamogeton sp.; Najas sp. & Alves and Gorni (2007) \\
\hline & Mayaca fluviatilis; Najas sp. & Gorni and Alves (2008) \\
\hline & Egeria $\mathrm{sp}$. & This study \\
\hline Pristina osborni (Walton, 1906) & Potamogeton sp. & Alves and Gorni (2007) \\
\hline \multirow{3}{*}{$\begin{array}{l}\text { Pristina proboscidea } \text { Beddard, } \\
1896\end{array}$} & Ipomoea $\mathrm{sp}$. & Alves and Gorni (2007) \\
\hline & Mayaca fluviatilis; Najas sp. & Gorni and Alves (2008) \\
\hline & Egeria sp.; Salvinia sp. & This study \\
\hline Pristina rosea (Piguet, 1906) & Ceratophyllum sp.; Egeria sp.; Utricularia sp. & This study \\
\hline Pristina synclites Stephenson, 1925 & Ceratophyllum sp.; Egeria $\mathrm{sp.}$ & This study \\
\hline Pristina sp. Ehrenberg, 1828 & Eichhornia azurea & $\begin{array}{l}\text { Saulino and Trivinho- } \\
\text { Strixino (2014) } \\
\end{array}$ \\
\hline \multirow{5}{*}{ Slavina evelinae (Marcus, 1942) } & Cabomba sp.; Potamogeton sp. & Alves and Gorni (2007) \\
\hline & Scirpus cubensis & $\begin{array}{l}\text { Correia and Trivinho- } \\
\text { Strixino (1998) } \\
\end{array}$ \\
\hline & Scirpus cubensis & $\begin{array}{l}\text { Trivinho-Strixino et al. } \\
(2000)\end{array}$ \\
\hline & Hydrilla verticillata; Egeria najas & Behrend et al. (2013) \\
\hline & Egeria sp.; Salvinia sp.; Ceratophyllum sp. & This study \\
\hline Slavina sp. Vejdovsky, 1883 & Eichhornia azurea & $\begin{array}{l}\text { Saulino and Trivinho- } \\
\text { Strixino (2014) } \\
\end{array}$ \\
\hline $\begin{array}{l}\text { Stephensoniana trivandrana } \\
\text { (Aiyer, 1926) }\end{array}$ & Eichhornia azurea & $\begin{array}{l}\text { Montanholi-Martins and } \\
\text { Takeda (2001) } \\
\end{array}$ \\
\hline \multirow{3}{*}{ Stylaria lacustris (Linnaeus, 1767) } & Egeria sp.; Cabomba sp.; Ipomoea sp.; Utricularia sp. & Alves and Gorni (2007) \\
\hline & Hydrilla verticillata & Behrend et al. (2013) \\
\hline & Egeria sp.; Salvinia sp.; Utricularia sp. & This study \\
\hline \multicolumn{3}{|l|}{ Opistocystidae } \\
\hline $\begin{array}{l}\text { Opistocysta funiculus Cordero, } \\
1948\end{array}$ & Ceratophyllum sp.; Egeria sp.; Eleocharis sp. & This study \\
\hline Opistocysta serrata Harman, 1970 & Eichhornia azurea & $\begin{array}{l}\text { Montanholi-Martins and } \\
\text { Takeda (2001) }\end{array}$ \\
\hline
\end{tabular}


TABLE 2: Macrophyte life forms; absolute (n) and relative (\%) number of Oligochaeta taxa associated with macrophytes (regarding 45 Oligochaeta taxa registered).

\begin{tabular}{lccc}
\hline \multicolumn{1}{c}{ Macrophytes } & Life forms & n & \% \\
\hline Eichhornia azurea & Floating & 23 & 51.1 \\
Egeria sp. & Submerged & 18 & 40.0 \\
Najas sp. & Submerged & 16 & 35.6 \\
Mayaca fluviatilis & Submerged & 14 & 31.1 \\
Salvinia sp. & Floating & 13 & 28.9 \\
Ceratophyllum sp. & Submerged & 12 & 26.7 \\
Eleocharis sp. & Submerged & 11 & 24.4 \\
Ipomoea sp. & Submerged & 11 & 24.4 \\
Utricularia sp. & Submerged & 11 & 24.4 \\
Potamogeton sp. & Submerged & 10 & 22.2 \\
Cabomba sp. & Submerged & 9 & 20.0 \\
Egeria najas & Submerged & 7 & 15.6 \\
Hydrilla verticillata & Submerged & 7 & 15.6 \\
Scirpus cubensis & Emergent & 7 & 15.6 \\
Cabomba piauhyensis & Submerged & 4 & 8.9 \\
Mayaca sp. & Submerged & 4 & 8.9 \\
Polygomum sp. & Emergent & 4 & 8.9 \\
Salvinia auriculata & Floating & 4 & 8.9 \\
Scirpus sp. & Emergent & 2 & 4.4 \\
Nymphaea sp. & Floating & 1 & 2.2 \\
\hline
\end{tabular}

\section{Discussion}

Our study provides an overview of the association between Oligochaeta and macrophytes in Brazilian continental aquatic environments. We highlighted the Naididae family, which obtained $93 \%$ of the species richness. Similarly, our results showed the Eichhornia azurea macrophyte with a higher richness of associated Oligochaeta species.

Regarding the Naididae family, perhaps the higher representativeness is due to the inclusion of recent subfamilies (Tubificinae, Rhyacodrilinae, Phallodrilinae, Limnodrilinae, and Telmatodrilinae) to the group (CHRISTOFFERSEN, 2007). However, this pattern is confirmed by the literature, which regards Naididae as the main family among the Oligochaeta (PAMPLIN et al., 2005).

Naididae is a group adapted to many environmental conditions and it has a wide distribution, all over the world. Moreover, these organisms are free swimmers, a feature which allows them to explore a broader diversity of habitats, including macrophytes (VERDONSCHOT et al., 1982; ALVES; GORNI, 2007). The main food source of these organisms consist of periphyton accumulated in organic matter on plants and sediment (BRINKHURST; JAMIESON, 1971; SCHENKOVÁ; HELESIC, 2006). Thus, some authors reported that the Naididae family is favored at sites with the presence of aquatic vegetation (LEARNER et al., 1978; SMOCK; STONEBURNER, 1980; ALVES; GORNI, 2007).

Our results revealed that Dero species obtained the highest prevalence in the macrophytes, they were observed in plants with different life forms: emergent, floating, and submerged, demonstrating the adaptive capacity of this group to various habitats. In this study, the species Dero nivea had the highest prevalence and it is associated to 15 macrophyte taxa. According to other studies, this species is commonly found in regions rich 
in aquatic vegetation (DAVIS, 1982) and it is usually one of the most abundant species (CORREIA; TRIVINHOSTRIXINO, 1998; TRIVINHO-STRIXINO et al., 2000; ALVES; GORNI, 2007). In turn, the species with lower prevalence, such as Brinkhurstia americana and Limnodrilus hoffmeisteri, are more adapted to live on sediment and the substratum composition and particle size was a major determinant of their distribution (MORETTO et al., 2013).

The three-dimensional complexity of macrophytes provides periphyton accumulation and, as a consequence, greater abundance and richness of Naididae, when compared to other aquatic invertebrates (THOMAZ; CUNHA, 2010; HANSEN et al., 2011). Moreover, plant position in the water column may affect the occurrence of organisms. Floating macrophytes, for instance, develop a dense root system, which provides many aquatic organisms with shelter (HECKMAN, 1998), including some Naididae species, which can build small tubes with root fragments, connected by tegument secretions (ARMENDÁRIZ, 2008). Thus, this structural complexity form slits that serve as hiding places and nests, reducing river flow (GREGG; ROSE, 1982) and protecting against predators (FERREIRO et al., 2011). Additionally, the Oligochaeta also occurred on exotic macrophytes, Hydrilla verticillata, which according to Behrend et al. (2013) provide these organisms with favorable conditions.

Eichhornia azurea, which had the highest richness of associated Oligochaeta species, is commonly found in studies associating macroinvertebrates to aquatic macrophytes (FULAN; HENRY, 2006; COPATTI et al., 2013; SILVA; HENRY, 2013). This species lives a floating life, providing organisms with spatial stability, it also has thick roots and submerged leaves, providing microhabitats to aquatic fauna (POTT; POTT, 2000). Among all features reported above, it is worth highlighting the submerged rhizome biomass, which absorb and store nutrients, keeping continued productivity and regular food sources to macroinvertebrates (OLIVEIRA et al., 2005). Other factors, such as the decomposition mechanisms of these macrophytes and release of particulate nutrients in aquatic environments may explain the high richness of Oligochaeta species registered for this macrophyte.

This study registered about $48 \%$ of the aquatic Oligochaeta diversity observed in Brazil, and this result highlights the relevance of macrophytes as a resource for these invertebrates, mainly for the Naididae family. The literature review also revealed a small number of studies conducted in Brazil associating Oligocheta to macrophytes. Thus, this study corroborates Christoffersen (2010), who reported that information on these worms in South America are regarded as insufficient and further investigation is needed to increase knowledge on the distribution and ecology of this taxon. Therefore, this article contributes to the collection of scientific data on the Oligochaeta in Brazilian continental aquatic environments.

\section{Acknowledgments}

The authors thank to the Brazilian Coordination for the Improvement of Higher Education Personnel (CAPES) and the Brazilian National Foundation for Development of Private Higher Education (FUNADESP) for their financial support.

\section{References}

ALVES, R. G.; GORNI, G. R. Naididae species (Oligochaeta) associated with submersed aquatic macrophytes in two reservoirs (São Paulo, Brazil). Acta Limnologica Brasiliensia, Rio Claro, v. 19, n. 4, p. 407-413, 2007.

ALVES, R. G.; MARCHESE, M. R.; ESCARPINATI, S. C. Oligochaeta (Annelida, Clitellata) in lotic environments in the state of São Paulo, Brazil. Iheringia, Série Zoologia, Porto Alegre, v. 96, n. 4, p. 431-435, 2006.

ALVES, R. G.; MARCHESE, M. R.; MARTINS, R. T. Oligochaeta (Annelida, Clitellata) of lotic environments at Parque Estadual Intervales (São Paulo, Brasil). Biota Neotropica, Campinas, v. 8, n. 1, p. 69-72, 2008.

ARMENDÁRIZ, L. C. Ciclo de vida de Dero (Aulophorus) costatus Marcus, 1944 (Tubificidae, Oligochaeta) en un cuerpo de agua com vegetation flotante en los Talas, Argentina. Gayana, Concepción, v. 72, n. 1, p. 23-20, 2008.

BEHREND, R. D. L.; TAKEDA, A. M.; GOMES, L. C.; FERNANDES, S. E. P. Using Oligochaeta assemblages as an indicator of environmental changes. Brazilian Journal of Biology, São Carlos, v. 72, n. 4, p. 873-884, 2012. 
BEHREND, R. D. L.; TEIXEIRA, M. C.; FERNANDES, S. E. P.; CAMARGO, J. C.; ROSIN, G. C.; TAKEDA, A. M. Effects of a native and a non-native macrophyte species of Hydrocharitaceae on Chironomidae and Oligochaeta assemblages structure. Acta Scientiarum, Maringá, v. 35, n. 3, p. 351-358, 2013.

BRINKHURST, R. O.; JAMIESON, B. G. M. Aquatic Oligochaeta of the world. Toronto: University of Toronto Press, 1971. $860 \mathrm{p}$.

BRINKHURST, R. O.; MARCHESE, M. R. Guia para la identificación de oligoquetos acuáticos continentales de Sud y Centroamerica. Santa Fé: Asociación de Ciencias Naturales del Litoral, 1989. 207 p.

CAMPITELLI-RAMOS, R.; LUCCA, J. V.; OLIVEIRA, L. L. D.; MARCHESE, M. R.; ROCHA, O. First record of Dero (Aulophorus) bimagnasetus Harman (Oligochaeta) from Brazil and habitat characteristics. Brazilian Journal of Biology, São Carlos, v. 74, n. 2, p. 483-488, 2014.

CHAPMAN, P. M. Utility and relevance of aquatic oligochaetes in ecological risk assessment. Hydrobiologia, Brussels, v. 463, p. 149-169, 2001.

CHAUVET, E. Leaf litter decomposition in large rivers: the case of the River Garonne. Limnetica, Barcelona, v. 13, n. 2, p. 65-70, 1997.

CHRISTOFFERSEN, M. L. A catalogue of aquatic microdrile oligochaetas (Annelida: Clitellata) from South America. Acta Hydrobiologica Sinica, Wuhan, v. 31, p. 59-86, 2007.

CHRISTOFFERSEN, M. L. Continental biodiversity of South American oligochaetes: the importance of inventories. Acta Zoológica Mexicana, Cidade do México, número especial 2, p. 35 46, 2010.

COPATTI, C. E.; FAGUNDES, L. S.; QUAINI, J. B.; COPATTI, B. R. Diversity of aquatic arthropods on Eichhornia crassipes (Mart.) Solms roots before and after removal of substrate in a reservoir in southern Brazil. Pan-American Journal of Aquatic Sciences, Rio Grande, v. 8, n. 4, p. 265-275, 2013.

CORBI, J. J.; GORNI, G. R.; CORREA, R. C. An evaluation of Allonais inaequalis Stephenson, 1911 (Oligochaeta: Naididae) as a toxicity test organism. Ecotoxicology Environmental Contamination, Itajaí, v. 10, n. 1, p. 7-11, 2015.

CORREIA, L. C. S.; TRIVINHO-STRIXINO, S. Macroinvertebrados da Rizosfera de Scirpus cubensis na lagoa do Infernão (Estação Ecológica de Jataí - SP): estrutura e função. Acta Limnologica Brasiliensia, Rio Claro, v. 10, n. 1, p. 37-47, 1998.

DAVIS, J. R. New records of aquatic Oligochaeta from Texas, with observations on their ecological characteristics. Hydrobiologia, Brussels, v. 96, p. 15-29, 1982.

DORNFELD, C. B.; ALVES, R. G.; LEITE, M. A.; ESPÍNDOLA, E. L. G. Oligochaeta in eutrofic reservoir: the case of Salto Grande reservoir and their main affluent (Americana, São Paulo, Brazil). Acta Limnologica Brasiliensia, Rio Claro, v. 18, n. 2, p. 189-197, 2006.

ESTEVES, F. A.; LEAL, J. J. F.; CALlisto, M. Comunidade bentônica. In: ESTEVES, F. A. (Ed.). Fundamentos de limnologia. 3 ed. Rio de Janeiro: Ed. Interciência, 2011. 790 p.

FERREIRO, N.; FEIJOÓ, C.; GIORGI, A.; LEGGIERI, L. Effects of macrophyte heterogeneity and food availability on structural parameters of the macroinvertebrate community in a Pampean stream. Hydrobiologia, Brussels, v. 664, n. 1, p. 199-211, 2011.
FULAN, J. A.; HENRY, R. The Odonata (Insecta) assemblage on Eichhornia azurea (Sw.) Kunth (Pontederiaceae) stands in Camargo Lake, a lateral lake on the Paranapanema River (state of São Paulo, Brazil), after an extreme inundation episode. Acta Limnologica Brasiliensia, Rio Claro, v. 18, n. 4, p. 423-431, 2006.

GALIZZI, M. C.; MARCHESE, M. Invertebrate colonization on Eucalyptus camaldulensis Dehnhardt leaf litter breakdown in na anabranche of the Middle Paraná River. Hidrobiológica, Cidade do México, v. 19, n. 2, p. 141-149, 2009.

GLOWACKA, I.; SOSZKA, G. J.; SOSZKA, H. Invertebrates associated with macrophytes. In: PIECZYNSKA, E. (Org.). Selected problems of lake littoral ecology. Warszawa: Widawnictwa Uniwersytetu Warszawskiego, 1976. 238 p.

GORNI, G. R.; ALVES, R. G. Naididae (Annelida, Oligochaeta) associated with Pomacea bridgesii (Reeve) (Gastropoda, Ampullaridae). Revista Brasileira de Zoologia, Curitiba, v. 23, n. 4, p. 1059-1061, 2006.

GORNI, G. R.; ALVES, R. G. Naididae (Annelida, Oligochaeta) associated with briophytes in Brotas, State of São Paulo, Brazil. Revista Brasileira de Zoologia, Curitiba, v. 24, n. 2, p. 518-519, 2007.

GORNI, G. R.; ALVES, R. G. Naididae species (Annelida: Oligochaeta) associated with the sponge Metania spinata (Carter, 1881) (Porifera: Metaniidae) from a southeastern reservoir. Acta Limnologica Brasiliensia, Rio Claro, v. 20, n. 3, p. 261-263, 2008.

GORNI, G. R.; ALVES, R. G. Oligochaetas (Annelida, Clitellata) in a neotropical stream: a mesohabitat approach. Iheringia, Série Zoologia, Porto Alegre, v. 102, n. 1, p. 106-110, 2012.

GORNI, G. R.; PEIRÓ, D. F.; SANCHES, N. Aquatic Oligochaeta (Annelida: Clitellata) from State of São Paulo, Brazil: Diversity and Ocurrence review. Biota Neotropica, Campinas, v. 15, n. 1, p. 1-8, 2015.

GREGG, W. W.; ROSE, F. L. The effects of aquatic macrophytes on the stream microenvironment. Aquatic Botany, Gainesville, v. 14, p. 309-324, 1982.

HANSEN, J. P.; WIKSTRÖM, S. A.; AXEMAR, H.; KAUTSKY, L. Distribution differences and active habitat choices of invertebrates between macrophytes of different morphological complexity. Aquatic Ecology, Heidelberg, v. 45, n. 1, p. 11-22, 2011.

HECKMAN, C. W. The seasonal succession of biotic communities in wetlands of the tropical wet-and-dry climatic zone: V. Aquatic invertebrate communities in the Pantanal of Mato Grosso, Brazil. International Review of Hydrobiology, Weinheim, v. 83, n. 1, p. 31-63, 1998.

HELLAWELL, J. M. Biological Indicator of freshwater pollution and environmental management. London: Elsevier Applied Science Publishers, 1986. 546 p.

KRAWCZYK, A. C. D. B.; BALDAN, L. T.; ARANHA, J. M. R.; MENEZES, M. S.; ALMEIDA, C. V. The invertebrates's community in adjacent Alto Iguaçu's anthropic lakes of different envorinmental factors. Biota Neotropica, Campinas, v. 13, n. 1, p. 21-34, 2013.

LEARNER, M. A.; LOCHHEAD, G.; HUGHES, B. D. A. review of the biology of the British Naididae (Oligochaeta) with emphasis on the lotic environment. Freshwater Biology, London, v. 8, p. 357-375, 1978. 
MAGURRAN, A. E. Medindo a diversidade biológica. Curitiba: Editora UFPR, 2011. 262 p.

MARTINS, R. T.; SILVEIRA, L. S.; ALVES, R. G. Colonization by Oligochaetas (Annelida: Clitellata) in decomposing leaves of Eichhornia azurea (SW.) Kunth (Pontederiaceae) in a neotropical lentic system. Annales de Limnologie - International Journal of Limnology, London, v. 47, p. 339-346, 2011.

MONTANHOLI-MARTINS, M. C.; TAKEDA, A. M. Spatial and temporal variations of oligochaetas of the lvinhema river and Patos lake in the upper Paraná river basin, Brazil. Hydrobiologia, Brussels, v. 463, n. 1-3, p. 197-205, 2001.

MORETTO, Y.; SIMÕES, N. R.; BENEDITO, E.; HIGUTI, J. Effect of trophic status and sediment particle size on diversity and abundance of aquatic Oligochaeta (Annelida) in neotropical reservoirs. Annales de Limnologie - International Journal of Limnology, London, v. 49, n. 1, p. 65-78, 2013.

MORMUL, R. P.; THOMAZ, S. M.; HIGUTI, J.; MARTENS, K. Ostracod (Crustacea) colonization of a native and a non-nativa macrophyte species of Hydrocharitaceae in the upper Paraná floodplain (Brazil): an experimental evaluation. Hydrobiologia, Brussels, v. 644, n. 1, p. 185-193, 2010.

ODA, F. H.; PETSCH, D. K.; RAGONHA, F. H.; BATISTA, V. G.; TAKEDA, A. M.; TAKEMOTO, R. M. Dero (Allodero) lutzi Michaelsen, 1926 (Oligochaeta: Naididae) associated with Scinax fuscovarius (Lutz, 1925) (Anura: Hylidae) from Semi-deciduous Atlantic Rain Forest, southern Brazil. Brazilian Journal of Biology, São Carlos, v. 75, p. 86-90, 2015.

OLIVEIRA, A. K. M.; FAVERO, S.; COSTACURTA, M. B. Variação temporal da biomassa de Eichhornia azurea (Sw.) Kunth (Pontederiaceae) e macrófitas aquáticas associadas em uma lagoa do Rio Negro, pantanal do Rio Negro, Mato Grosso do Sul. Natureza On Line, Santa Tereza, v. 3, n. 1, p. 7-12, 2005.

PAMPLIN, P. A. Z.; ROCHA, O.; MARCHESE, M. Riqueza de espécies de Oligochaeta (Anellida, Clitellata) em duas represas do Rio Tietê (São Paulo). Biota Neotropica, Campinas, v. 5, n. 1, p. 1-8, 2005.

PEIRÓ, D. F.; GORNI, G. R. Diferença na composição da entomofauna aquática associada a duas plantas de hábitos diferentes em um tanque de psicultura. Multiciência, Campinas, v. 10, p. 149$160,2010$.

PETSCH, D. K.; PINHA, G. D. ; RAGONHA, F. H. ; TAKEDA, A. M. Influência dos fatores ambientais sobre a distribuição da comunidade de invertebrados bentônicos em canais de uma planície de inundação neotropical. Biotemas, Florianópolis, v. 26, p. 127$138,2013$.

PETSCH, D. K.; RAGONHA, F. H.; GIMENEZ, B. C. G.; BARBOZA, L. G. A.; TAKEDA, A. M. Beta diversity partitioning of aquatic Oligochaeta in different environments of a neotropical floodplain. Acta Scientiarum, Maringá, v. 37, p. 41-49, 2015.

POTT, V. J.; POTT, A. Plantas aquáticas do Pantanal. Brasília: Embrapa - Centro de Pesquisa Agropecuária do Pantanal, 2000. $404 \mathrm{p}$.
RIGHI, G. Manual de identificação de invertebrados límnicos do Brasil. Brasília: CNpq, 1984. 48 p.

RODRIGUES, L. F. T.; LEITE, F. S.; ALVES, R. G. Inventory and distribution of Oligochaeta (Annelida: Clitellata) in first-order streams in preserved áreas of the state of Minas Gerais, Brazil. Biota Neotropica, Campinas, v. 13, n. 1, p. 245-254, 2013.

ROSINE, W. N. The distribution of invertebrates on submerged aquatic plant surfaces in Muskee Lake, Colorado. Ecology, New York, v. 36, p. 308-314, 1955.

SAULINO, L. H. H.; TRIVINHO-STRIXINO, S. Macroinvertebrados aquáticos associados às raízes de Eichhornia azuera (Swarts) Kunth (Pontederiaceae) em uma lagoa marginal no Pantanal, MS. Biotemas, Florianópolis, v. 27, n. 3, p. 65-72, 2014. SCHENKOVÁ, J.; HELESIC, J. Habitat preferences of aquatic Oligochaeta (Annelida) in the Rokytná River, Czech Republic - a small highland stream. Hydrobiologia, Brussels, v. 564, n. 1, p. 117-126, 2006.

SILVA, C. V.; HENRY, R. Aquatic macroinvertebrates associated with Eichhornia azurea (Swatrs) Khunt and relationships with abiotic factors in marginal lentic ecosystems (São Paulo, Brazil). Brazilian Journal of Biology, São Carlos, v. 73, n. 1, p. 149-162, 2013.

SILVEIRA, L. F.; BEISIEGEL, B. M.; CURCIO, F. F.; VALDUJO, P. H.; DIXO, M.; VERDADE, K. V.; MATTOX, G. M. T.; CUNNINGHAM, P. T. M. Para que servem os inventários de fauna? Estudos Avançados, São Paulo, v. 24, n. 68, p. 173-207, 2010.

SMOCK, L. A.; STONEBURNER, D. L. The response of macroinvertebrates to aquatic macrophyte decomposition. Oikos, Copenhagen, v. 35, n. 3, p. 397-403, 1980.

THOMAZ, S. M; CUNHA, E. R. The role of macrophytes in habitat structuring in aquatic ecosystems: methods of measurement, causes and consequences on animal assemblages composition and biodiversity. Acta Limnologica Brasiliensia, Rio Claro, v. 22, n. 2, p. 218-236, 2010.

TRIVINHO-STRIXINO, S.; CORREIA, L. C. S.; SONODA, K. Phytophilous Chironomidae (Diptera) and other macroinvertebrates in the ox-bow Infernão lake (Jataí Ecological Station, Luiz Antônio, SP, Brazil). Revista Brasileira de Biologia, São Carlos, v. 60, n. 3, p. $527-535,2000$.

TRIVINHO-STRIXINO, S.; GESSNER, A. F.; CORREIA, L. Macroinvertebrados associados a macrófitas aquáticas as lagoas marginais da Estação Ecológica do Jataí (Luiz Antônio SP). In: SEMINÁRIO REGIONAL DE ECOLOGIA DO PPGERN/ UFSCar, VIII, 1997, São Carlos. Anais... São Carlos: PPGERN/ UFSCar, 1997, v. 8, p. 53-60.

VERDONSCHOT, P. F. M.; SMIES, M.; SEPERS, A. B. J. The distribution of aquatic oligochaetes in brackish inland waters in the SW Netherlands. Hydrobiologia, Brussels, v. 89, p. 29-38, 1982.

WELCH, P. S. Limnology. 2 ed. New York: McGraw-Hill, 1952. $538 \mathrm{p}$. 